\title{
Material ecologies for synthetic biology: Biomineralization and the state space of design
}

\author{
Martyn Dade-Robertson ${ }^{\mathrm{a}, *}$, Carolina Ramirez Figueroa ${ }^{\mathrm{a}}$, Meng Zhang ${ }^{\mathrm{b}, 1}$ \\ ${ }^{\text {a } A r c h a I D, ~ S c h o o l ~ o f ~ A r c h i t e c t u r e ~ P l a n n i n g ~ a n d ~ L a n d s c a p e, ~ N e w c a s t l e ~ U n i v e r s i t y, ~ N e w c a s t l e ~ u p o n ~ T y n e, ~ N E 1 ~ 7 R U, ~ U K ~}$ \\ ${ }^{\mathrm{b}}$ Cellular \& Molecular Sciences Group, Faculty of Health and Life Sciences, Northumbria University, Newcastle upon Tyne, NE1 8ST, UK
}

\section{H I G H L I G H T S}

- Synthetic Biology requires understanding physical materials as computational agents.

- Biological materials may not be produced but induced.

- Biological state spaces include cell, and chemical and physical constraints.

- Synthetic Biology represents a new opportunity for material ecologies.

\section{A R T I C L E I N F O}

\section{Keywords:}

Synthetic biology

Material ecologies

Self assembly

Emergence

State space

\begin{abstract}
A B S T R A C T
This paper discusses the role that material ecologies might have in the emerging engineering paradigm of Synthetic Biology (hereafter SB). In this paper we suggest that, as a result of the paradigm of SB, a new way of considering the relationship between computation and material forms is needed, where computation is embedded into the material elements themselves through genetic programming. The paper discusses current trends to conceptualize SB in traditional engineering terms and contrast this from design speculations in terms of bottom-up processes of emergence and self-organization. The paper suggests that, to reconcile these positions, it is necessary to think about the design of new material systems derived from engineering living organisms in terms of a state space of production. The paper analyses this state space using the example of biomineralization, with illustrations from simple experiments on bacteria-induced calcium carbonate. The paper suggests a framework involving three interconnected state spaces defined as: cellular (the control of structures within the cell structures within a cell, and specifically DNA and its expression through the process of transcription and translation); chemical (considered to occur outside the cell, but in direct chemical interaction with the interior of the cell itself); physical (which constitutes the physical forces and energy within the environment). We also illustrate, in broad terms, how such spaces are interconnected. Finally the paper will conclude by suggesting how a material ecologies approach might feature in the future development of SB.
\end{abstract}

(c) 2014 Elsevier Ltd. All rights reserved.

\section{Introduction}

Whereas the latter part of the twentieth century was transformed through information technologies, it is widely predicted that the 21st century world will be radically changed by the emergence of biotechnology. Biological systems exhibit a wide variety

\footnotetext{
* Corresponding author. Tel.: +4401912085926.

E-mail addresses: martyn.dade-robertson@ncl.ac.uk (M. Dade-Robertson) p.c.ramirez-figueroa@newcastle.ac.uk (C. Ramirez Figueroa), meng.zhang@unn.ac.uk (M. Zhang).

14401912274218.
}

of forms and functions, make highly efficient use of energy and other resources, and are capable of processes such as programmed self-assembly and adaptability which are very difficult or, in some cases, impossible to achieve using more traditional human engineered systems. To this end, Synthetic Biology (hereafter SB) has been heralded as an important technological and design paradigm, enabling the development of complex material systems. SB is, however, still regarded as an emerging engineering discipline [1] and has yet to realize its full potential in terms of delivering complex synthetic biological systems.

In this paper we suggest that, as a result of the paradigm of SB, a new way of considering the relationship between computation and material forms is needed, where computation is 
embedded into the material elements themselves through genetic programming. The theme of material ecologies proposes a tight coupling between computational simulation, material properties and the manufacture of material form. Furthermore, it is proposed that design methods developed in the context of material ecologies may find, in a modified form, new applications for the creation of biological materials which are manufactured by living cells.

This paper analyses the relationship between SB, material design and production through first, in Part Two, examining how SB can be approached as an engineering design discipline, and then contrasting this with more speculative discourses in design. This paper will then seek to rationalize these two apparently contradictory approaches by examining the distinction between emergence, self-organization and the state space of material assembly.

Part Three will go on to describe the results of an experiment into biomineralization, the process by which living organisms induce the formation of mineral crystals. The experiment will be used to illustrate complexity in the creation of biological materials by showing the interrelationships between the living organism and its chemical and physical environment. This experiment, which observes the process by which bacterial communities induce biomineralization, will be used to develop a design framework which describes biomineralization as a process of material fabrication and assembly. This framework is illustrated by proposing the design of a synthetic shell by controlling aspects of the state space of material production in the context of a biological system. The outcomes from a further, limited, experiment will be used to demonstrate the effect of such a state space intervention on different scales of material fabrication and assembly.

Finally the paper will conclude by suggesting how a material ecologies approach might feature in the future development of SB.

\section{Synthetic biology in engineering and design}

While SB is a new discipline (significant citations to the subject first appear in 2003 [2]) there is now a thriving debate on its status as a field of engineering and, more broadly, as a design discipline [1]. There are, however, two contrasting notions of SB which will be the focus here.

\subsection{Definitions of synthetic biology in science and engineering}

The purpose of SB, according to the Royal Academy of Engineering, is "to design and engineer biologically based parts, novel devices and systems as well as redesigning existing, natural biological systems" [3, p. 6]. SB is associated with molecular level manipulation of, often simple, organisms through genetic modification. Since the discovery of the structure of Deoxyribonucleic acid (hereafter DNA) by Watson and Crick in the 1950s, and the associated development of recombinant DNA in the 1970s, the knowledge of the role of DNA in defining the properties of cells, and thus the characteristics of all living organisms, has grown rapidly. Through the scientific discipline of Systems Biology we now have detailed descriptions of the mechanisms through which DNA is transcribed into messenger Ribonucleic acid (hereafter mRNA) to guide the production of protein molecules in protein factories called ribosomes. These proteins act as machines which perform all the functions of living cells. We can now map entire genomes for individual organisms and, increasingly, we are able to make associations between specific DNA expression and the characteristics and behaviors of individual organisms.

While DNA is often described as containing the blueprint of life, the relationship between DNA sequences, the expression of proteins and the characteristics of biological systems are significantly more complex than this analogy implies. While there are instances of isolated gene sequences resulting in clearly defined characteristics in an organism, much of what we understand in terms of the morphology and behavior of biological systems is derived from groups of different genes being expressed through the more complex (compared to the genome) proteome-the entire population of proteins produced by a cell or organism at particular growth stages or in particular environments [4]. This means that Systems Biology is a science of complexity, and reverse-engineering the relationship between gene expression and their function within an organism is very difficult. Where clear relationships between gene expression and protein function are known, however, recombinant DNA techniques can be used to, literally, cut and paste sequences of DNA from one organism into another, and for the new host organism to be modified by the expression of its new genes. A widely publicized example of this is the transplantation of a gene from bioluminescent jellyfish into mouse DNA to highlight the presence of certain proteins in mice bodies [5].

This process of genetic engineering offers a broad definition of SB which is associated with the practices of copying DNA sequences from the genome of organisms and, through recombinant DNA, importing the sequence from one organism to another, so that the host organism exhibits some characteristic of the donor. These practices are now routinely used within molecular biology laboratories and there are already wide applications of genetically modified organisms. There is, however, a stricter definition of SB being proposed, which is based on a more formal conceptualization of genetic modification as an engineering design process. Biological Systems, in this new context, are considered to be akin to electrical systems with biological circuits [6,7].

SB has become subject to wide ranging speculation in many fields of design through initiatives such as the Synthetic Aesthetics project $[8,9]$. SB has, however, been predominantly developed through collaboration between molecular biology and computer science. A highly influential approach to SB is emerging, which conceptualizes biological systems in terms of the design of more traditional forms of engineering. The emphasis of this approach is to simplify the process of designing biological systems by:

1. Engaging with an engineering design cycle which includes a clear set of requirements, design, implementation, testing, verification and refinement with an emphasis on extensive simulation and modeling throughout the process [6].

2. Describing DNA sequences and their products as standardized, self-contained parts which are interchangeable and can be used to construct genetic circuits for different functions [7].

3. Bypassing complex lab-based practices of recombinant DNA by using synthesized DNA(DNA which has been coded and 'printed out' from a computer).

This framework has shown early successes, notably by enabling a new generation of synthetic biologists to share and build upon each others' work through competitions such as iGEM, and its sister Repository of Standard Biological Parts. As the repository of parts grows, it is suggested that more complex biological systems will be possible through the assembly of many parts [10].

This approach to the design of biological systems is, however, contested. Some suggest that complex biological networks cannot be reduced and partitioned into discrete parts [11] and this leads to informal and often hidden SB practices. O'Malley [12], for example, suggests that 'While engineering certainly contributes to the practices of synthetic biology, (...) it is doing this in more complicated ways than might be envisioned in the "pure" engineering ideal'. O'Malley goes on to suggest than hidden behind public descriptions of SB are processes which could be characterized as Kludging (i.e. klumsy, lame, ugly, dumb, but also good enough). These practices, she argues, far from being a sign of failure should be seen as a "highly creative and effective process'. 


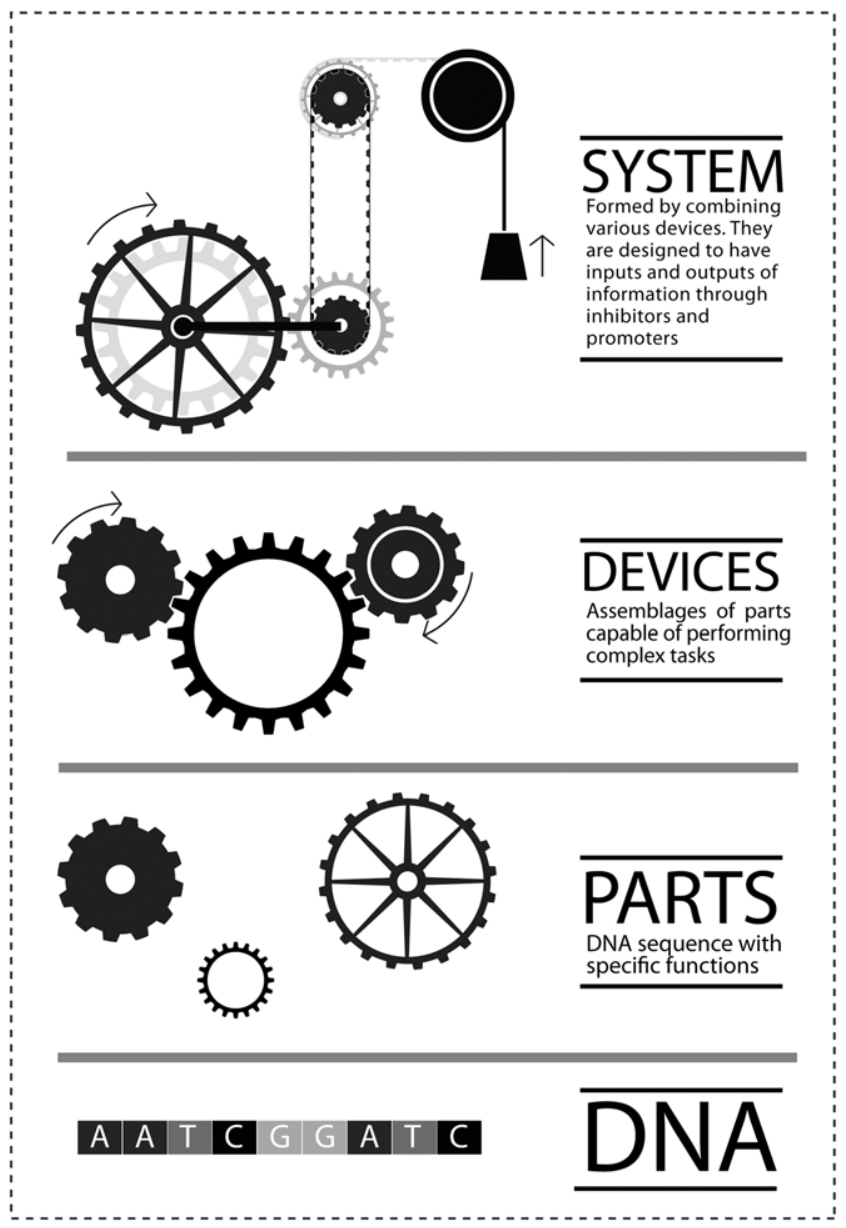

Fig. 1. The 'stack' model of SB based on a hierarchy of assembly from a single DNA sequence to a full system.

This debate about the nature of biological processes is reminiscent of a long-standing argument between elementalism (studying systems through their reduction into atom-like parts) and organicism (the study of the system as an irreducible whole by recognizing the role of emergent complexity) $[4,13,14]$. In SB, an elementalist model would emphasis DNA as the site for design intervention. An organicist view, however, may require new designs to be considered from multiple possible perspectives and involve the manipulation of chemical, physical and cellular environments in concert with possible design outcomes which are emergent and difficult to describe with reference to the functional parts alone (see Fig. 1).

\subsection{Definitions of synthetic biology in architectural design}

In the context of science and engineering the dominant discourse in SB, as described above, understands engineering biological systems as being akin to engineering mechanical or electronic systems with clear definitions of parts and their assembly, which can be expressed through functional hierarchies and, in turn, enable abstraction and top-down specification. However, in other design discourses, biological systems are deliberately distinguished from mechanical systems and from traditional methods of design and material articulation. The discourse on material ecologies, for example, deliberately seeks to understand engineered systems in nonlinear terms through a design process which considers factors such as material performance, design intent, fabrication and environment as reciprocal and interrelated. For example, in Oxman et al.'s work on material computation, materials are described though surfaces of 'continuous components' such as tiles and voxels, which represent cells and constitute both the material assemblage and processing units that adapt to inputs from their environment [15]. This material model, which is based on observations of real biological systems, acknowledges that material organization in living systems is not designed but, rather, emerges from constraints inherent in the materials themselves, and in their interaction with the environment. This approach has been taken even further in a design discourse which posits a 'literal biological paradigm', suggesting that the designer (specifically the architectural designer in this case) should 'go beyond using shallow biological metaphors or a superficial biomorphic formal repertoire' and, through architectures of synthetic life, understand the built environment as 'a synthetic life-form embedded within dynamic and generative ecological relations' [16, p. 18]. Furthermore, speculative architectural designers [17-19] have suggested that a literal biological paradigm alters the relationship between representation and designed object, enabling a design process more akin to cultivation than engineering.

When compared with the engineering approaches described above there is, we argue, the potential to considerably extend our current understanding of SB through approaches which recognize bottom-up and top-down approaches to the development of material systems.

\subsection{Emergence, self-organization and the state space of design}

The mechanism by which biological systems organize and build matter is generally understood as being 'bottom up'. This approach can be contrasted to 'top-down' human constructions, which tend to realize material form following a hierarchical and linear pattern of information and decision making. Examples of this can be found in architectural design processes, which follow a series of steps that go from spatial design, to detail design to material assembly. In $\mathrm{SB}$, however, the loose notion of 'bottom up' is unhelpful in that it hides the importance of temporal and spatial scales in biological systems. In the context of this study, it is worth distinguishing between emergence, programmed self-organization and the state space of design.

While both emergence and self-organization can occur at the same time, (indeed emergence is most often seen as a form of self-organization), a distinction between both terms has been developed by Wolf et al. where:

A system exhibits emergence when there are coherent emergents at the macro-level that dynamically arise from the interactions between the parts at the micro-level. Such emergents are novel... (with regard to)... the individual parts of the system [20, p. 3].

And self-organization is:

[...] a dynamical and adaptive process where systems acquire and maintain structure themselves, without external control (p. 7).

Both emergence and self-organization occur together throughout biological systems. Examples include the flocking patterns of birds and can be illustrated through simple algorithms such as the Game of Life, where the states of cells within a matrix, either dead or alive, are determined by following simple rules which simulate the effects of cell reproduction and death based on the proximity of local cells. While the rules governing the state of individual cells may be simple, the resulting dynamic patterns are complexleading to distinctive patterns in the clusters of cells which, for example, appear to glide across the screen or devour other clusters. It is, however, possible to have a system which is self-organizing but not emergent, being derived from a requirement to replicate 
particular forms and patterns. The work of Griffiths, for example, shows how a series of plastic laser cut tiles, given magnetic edges and fed through a channel onto an air table, can self-assemble into basic shapes (in the demonstration this consisted of the letters M, I and T) [21,22]. Even when the desired state of the system is a result of the interaction of individual agents, it cannot be considered emergent as the design pattern is preconfigured. Yet the elements are self-assembled under the logic presented by Wolf et al. in that the behavior of the parts has been restricted 'to confine it to a smaller volume of its state space' (7). Specifically, such systems represent programmed self-assembly in that the designer controls both the information contained in the system (in the Griffith example, the ways in which the tiles can joint to one another) and the space of interaction to achieve a desired and pre-designed outcome (for similar, three-dimensional approaches see [23]).

Biological systems can be considered to be emergent in terms of their evolution, i.e. the process of variation and natural selection by which simple biological structures have evolved to become more complex over long periods of time and multiple generations. Taken out of the context of deep time, however, the development of an organism, whether plant or animal, appears to follow defined developmental paths leading to predictable patterns with variations within confined boundaries, unless subject to significant mutations. Biological organisms can, therefore, be considered to be operating and finding form on the basis of programmed self-assembly where the code is written through an emergent and evolutionary process. The state space of biological material construction is one of developmental constraints defined by both the organism and its environment. More accurately, as shown below, biological materials are produced through the interaction of a number of different state spaces.

\section{The state space of biomineralization}

In real biological systems we can consider the state space of material construction to be, broadly, the interaction between cells and their environment. However, whilst in material-based computation, as described above, the unit of computation (through which an environment is sensed) and the unit of material are considered to be the same, in some instances of biological material production this is not the case. In the process of biomineralization, for example, the cell induces material through a fabrication process which is performed outside the cell and is, to some extent, independent of it. For this reason, and because of the importance of biomineralization throughout the biological world, we suggest that understanding biomineralization gives insight into the state space of biological material construction.

To help frame the argument, it is worth considering the case of abalone shells. Shells occupy an iconic status within design and have been used as an emblem of scale and proportion, as well as providing the material basis of many types of building materials. In addition, shell formation is a good example of the complex relationship between biological mechanisms, and the construction of inorganic materials through organic processes. An abalone constructs its shell by first forming soft tissues through morphogenesis (the process by which the organism's cells organize into tissue patterns). The organism does not build its shell directly. Instead, the tissues act like a scaffold that makes it possible for the abalone to alter the chemical composition of its outer surface, inducing calcium in its environment to combine with carbon and crystallize through the process of biomineralization. Furthermore, by altering the chemical environment through the addition of extra cellular substances, the shell shifts between different crystalline structures from the inner layer of flat plate-like crystals (sometimes referred to as mother of pearl) to the outer layer which is made up of vertical crystals that create an exceptionally strong surface [24]. By controlling this process, the abalone assembles a single material with properties of a composite material and creates a structure with significant strength with very little expenditure of energy.

\subsection{Biomineralization}

Biomineralization occurs in a wide range of processes and organisms, from the formation of calcium phosphate bone in mammals to calcium carbonate shells and teeth. Nature's harnessing of mineralization processes enables the creation of hard structures, which provide properties not found in soft tissue with low amounts of energy and maintenance [25].

In many organisms, biomineralization is a highly refined process derived from both chemical and physical control of crystal nucleation and morphology, which creates intricate patterns and structural variants. Resultant structures exhibit the properties of functionally graded materials, despite being virtually chemically homogeneous. As noted above, for example, abalone shells are composed of two distinct layers of calcite-based substances with a structurally robust outer 'Prismatic' layer, and a smooth but brittle internal 'Nacreous' layer. While derived from the same compound, these two layers have different crystalline structures and orientations and there is evidence to suggest that, through the release of extra cellular materials, abalones control the nucleation of the crystals, as well as their orientation, size, and density [26]. It is important to recognize, however, that the shell and its composite structure is the result of processes which are, to some extent, induced by the organism rather than produced (i.e. as the result of an extra cellular reaction between the organism and its environment).

Similar processes can be observed in a range of organisms including simple, single-celled bacteria. A number of bacteria strains, notably for this study, Bacillus pasteurii and Bacillus megaterium are known to change the conditions in a calcium-rich environment such that calcium carbonate is crystallized. These bacteria live extensively in soils and have been studied in the context of soil processes [27] and, along with calcium carbonate inducing bacteria, are thought to have created the earliest form of fossils through the remains of microbial mats known as stramatolites [28]. Bacterially induced $\mathrm{CaCO}_{3}$ has already been explored in the context of built environment research, specifically in 'self-healing' concrete [29], a product that uses bacteria buried in concrete aggregate to fill cracks in the material when exposed to air. The possibility of using modified bacteria to repair existing concrete has also been explored in Newcastle University's Gold Medal winning entry to MIT's iGEM competition in 2010. Called BacillaFilla, the team's proposal was to engineer bacteria to swim into and repair cracks in existing concrete structures [30].

This background of research into bacteria-induced calcium carbonate formation, combined with the relative simplicity of bacteria, makes them ideal as the basis for observing biomineralization and speculating about design possibilities using SB. Because biomineralization is already important in the production of building materials, there are clear applications that harness this process in both the remediation of existing materials and, potentially, the creation of new ones with little expenditure of energy. Whilst the applications described above have had relatively little control of the crystallization process itself, given the importance of crystal morphology and configuration to the mechanical properties of, for example, a shell, it is a reasonable hypothesis to suggest that by mastering crystal growth and development we will be able to create materials with variable properties which may be, for example functionally graded whilst being chemically homogeneous. Using 

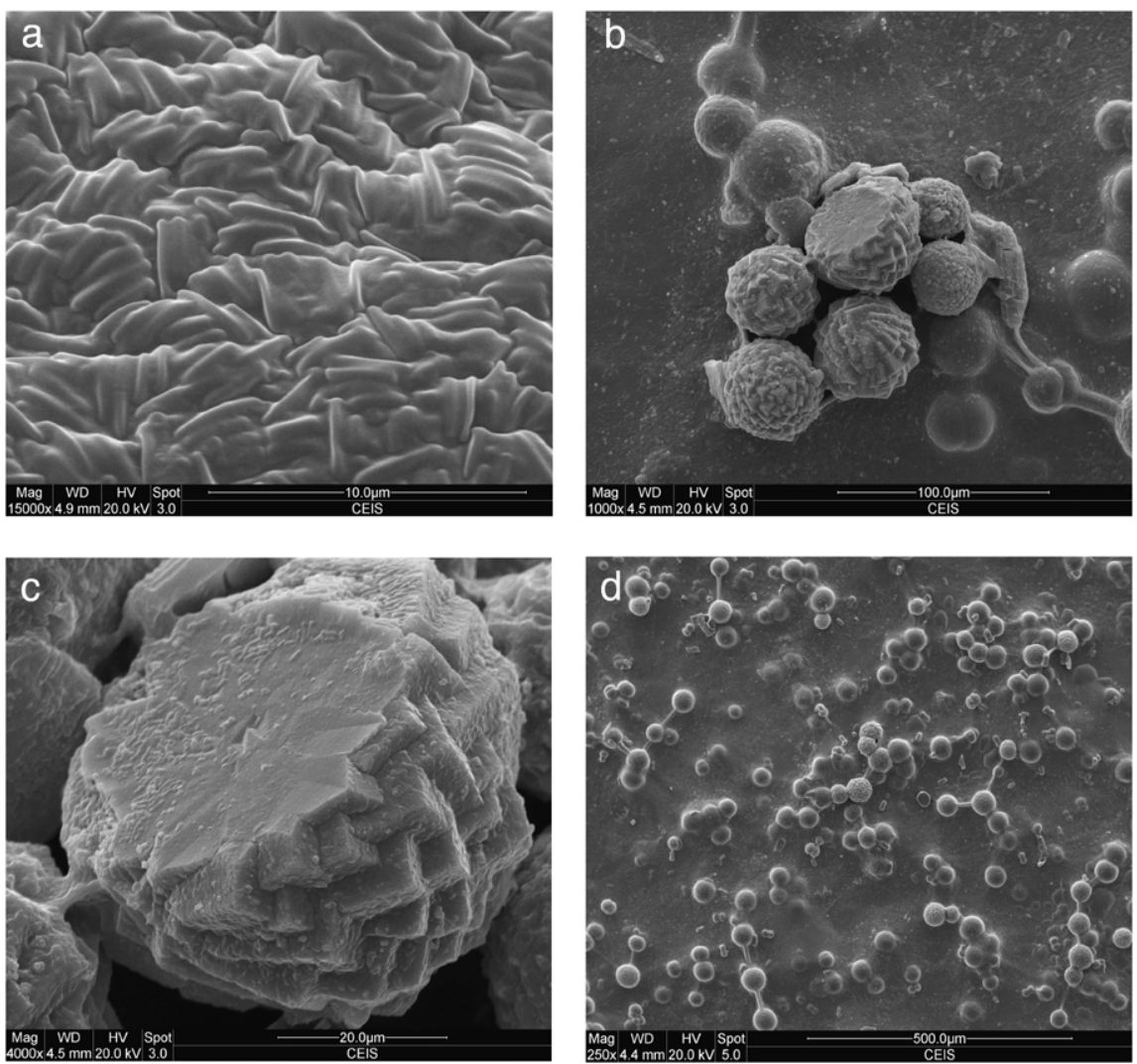

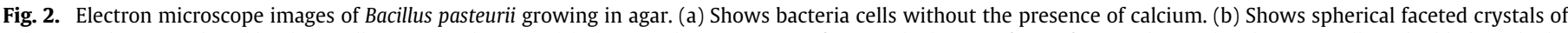

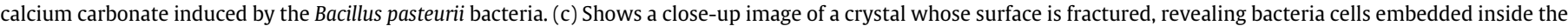
crystal structure. (d) Shows the network of fine filaments which connect the crystals together.

SB to achieve this would require being able to harness the biomineralization of simple, single-celled organisms such as bacteria to exhibit similar functions to much more complex organisms. Our experiments take this as a starting point and observe that the process of biomineralization using bacteria induce very small amounts of calcium carbonate.

The core role of bacteria in inducing calcium carbonate formation is straightforward. Within bacterial colonies, $\mathrm{CaCO}_{3}$ formation is usually initiated by the release of Urease, which is a by-product of bacterial metabolic activity [27]. Urease hydrolyzes the urea within the environment leading to an increase in $\mathrm{pH}$, which causes any calcium molecule to bind with carbon, triggering the formation of crystals at nucleation sites within the influence of bacterial colonies [31].

The function of bacteria in controlling biomineralization is, however, more complicated. Bacteria often form biofilms when they attach to a surface. Biofilms not only contain individual cells but also abundant extracellular structures known as extracellular polymeric substances (hereafter EPS) produced by the cells which contain DNA, proteins and polysaccharide. This helps the bacteria to adhere to surfaces and survive extreme external environments, as well as forming resistance against antibiotics. Within the biofilm architecture, there are chemical diffusion processes which allow the bacteria to metabolize, communicate with one another, and specialize across the colony. These structures can be found at scales from micrometers (tooth plaque for example) to tens of centimeters (in sewage flock for example).

\subsection{Experiment 1: bacterial biomineralization: comparing two bacte- ria strains}

Bacteria provide a useful starting point for the study of biomineralization. While bacteria are much simpler than multicellular organisms, the process of biomineralization induced by bacterial cells is similar to that found in more complex assemblages such as, for example, abalone shells [32,33]. Similarly, the effect of the EPS is much easier to observe and analyze in bacteria. For these reasons, bacteria are often used as a way of understanding the basic mechanisms of biomineralization in more complex organisms.

The basic hypothesis being tested by the initial experiment was that there would be significant differences in the material morphologies produced by different biologically induced conditions. This was tested using a series of simple microbiology experiments, in which bacteria were incubated in conditions which were conducive to biomineralization. In these experiments, we used two Bacillus species, i.e. B. pasteurii and B. megaterium, to show the different types of material structure resulting from different bacteria species within the same genus.

Growth medium was prepared using a recipe modified from [27], and then solidified by mixing with agar (a gelatin-like substance derived from seaweed). As control, samples of the two bacterial species were grown on agar plates containing rich nutrients and urea without calcium chloride. Under this condition, both bacteria achieved normal growth, but no calcium carbonate was produced. To observe the formation of calcium carbonate crystals, the Bacillus samples were grown on agar plates containing rich nutrients, urea and a high concentration of calcium chloride. Bacterial samples were streaked onto agar sample plates in three sections. Cultures were then incubated at a temperature of $30^{\circ} \mathrm{C}$ and, in the case of each of the bacteria, precipitation could be observed after a $24 \mathrm{~h}$ growth period.

Fig. 2(a) shows an electron microscope image of the control sample inoculated with $B$. pasteurii. The image shows thriving colonies of bacteria, seen here as long tubular cells, with no sign of crystal formation. Contrasting this to Fig. 2(b), we see that the 

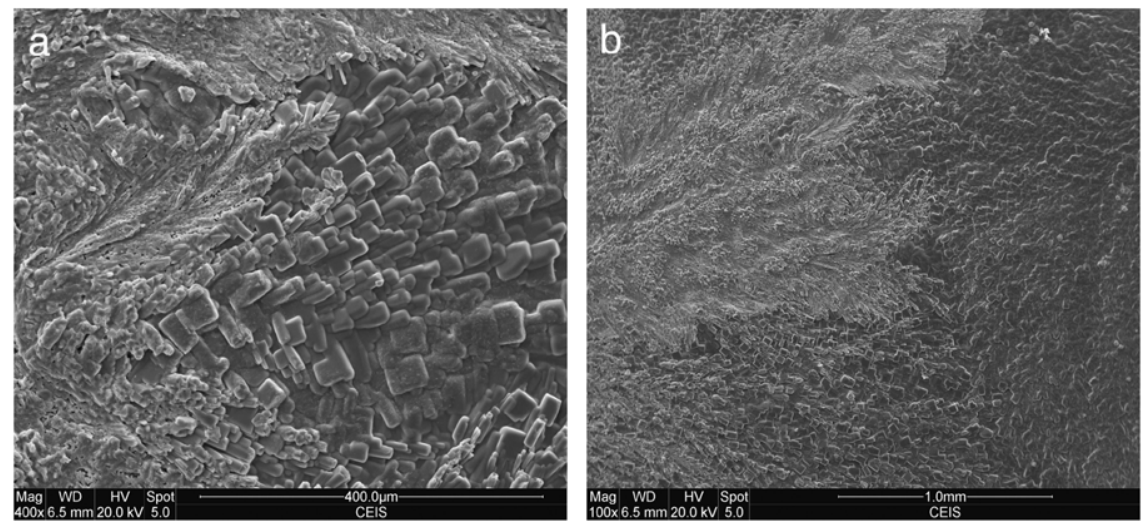

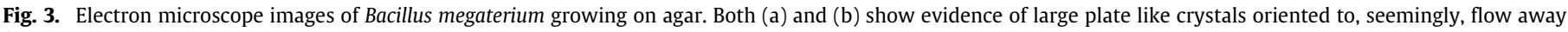
from the bacteria colony.

B. pasteurii culture grown using both urea and calcium chloride shows large, apparently multifaceted, spherical calcium carbonate crystals. This structural form is unique to calcium carbonate which has been crystallized in an environment rich in complex organic compounds [34]. Fig. 2(c) shows the same sample and makes evident the variation in scale between the bacterial cells and crystal. In this image, bacterial cells can be observed embedded into the surface of the crystal. It should also be noted that precipitation was observed across the whole plate.

A striking feature of the $B$. pasteurii sample is the structures which connect the crystals through a network of, what seem to be, fine filaments (Fig. 2(d)). Further analysis of the filaments reveals they are not crystalline structures, and it seems likely (although further analysis will need to be carried out to confirm this) that they are composed of organic molecules. The nature and purpose of these filaments is not yet clear, but given their regularity and spatial relationship to the crystals, it seems plausible to suggest that they are related to crystal nucleation and growth, and are a visible part of the biofilm. It is striking that, in this B. pasteurii sample, the influence of the bacteria spreads beyond the colony itself and crystals are found across the whole plate. It is therefore possible that this network structure is extending the influence of the bacteria beyond its immediate environment.

The $B$. pasteurii sample provides evidence of both unique crystal morphology and a biofilm structure which seems to interact with the induced materials. The $B$. megaterium experiment, on the other hand, produced much simpler structures (Fig. 3(a) and (b)). Crystals are flat and seem to follow the flow of the bacterial growth patterns, with no indication of a higher level structure. This lends support to the theory that the network of filaments found in the first sample was due to a reaction between the forming crystals and the extra cellular matrix of the bacteria, rather than to the agar substance. The $B$. megaterium sample also showed that the calcium carbonate only formed in very close proximity to bacterial colonies, which helps support the view that the network effect of the previous samples is not an artifact or a natural chemical process in the agar. While the basic process in both samples was the same, the radical difference in crystal morphology, distribution and the appearance of other structures indicates the importance of extracellular processes. These findings are backed up by others, for example [34], who have shown how the presence of EPS within a calcium carbonate crystallization environment can alter the characteristics of crystals, and substantially affect their morphology. Broadly speaking, differences in crystal morphology occur because of the presence and different concentrations of xanthan and amino acids. Experiments have also shown a direct relationship between cell growth and the presence of capsular polysaccharides (which compose a structural component of biofilms) [35] which may also help explain the structural network patterns found in our $B$. pasteurii sample. One of the reasons for this effect is likely to be that complex molecules found in the EPS bind to the surface of the crystal, either prompting or inhibiting crystal formation across specific faces of the crystal over specific times.

\subsection{State spaces in biomineralization}

The results presented in the experiments described above are far from conclusive. However, the patterns and processes observed do inform an understanding of material fabrication in simple biological systems. First, the experiments highlight an ambiguity in the production of biological materials because the process of crystal formation cannot be attributed to the bacteria alone. We cannot say that the bacteria directly produce calcium carbonate crystals. Rather, bacteria influence their environment through the release of compounds which induce calcium carbonate formation. However, even this account is limited. Mineral crystals form as a by-product of cell processes but, as has been shown in these experiments, the nucleation, extent and morphology of the crystals is unique to different bacteria. These different forms may be an evolutionary accident (it is not clear what purpose biomineralization has in the context of communities of bacteria) but they provide a clue to more complex biomineralization processes produced by other multicellular, organisms. In the study of material computation, a cell is responsive to its environment (able to sense and adapt to inputs) and also constitutes the material assemblage itself. However, in biomineralization the cell is a factory for the production of proteins which ultimately induce the formation of a material through their interaction with very specific environmental conditions. The minimal material unit in a bioinduced mineralization process might therefore be considered to be the calcium carbonate crystal or the DNA sequences responsible for encoding the production of urease. To understand biological material formation, the process needs to be understood in terms of different state spaces of material organization. Based on the experiment described above, we can define three interrelated state spaces of material construction: Cellular, Chemical and Physical spaces.

The cellular state space refers to processes which take place from within the cell within a cell-specifically DNA and its expression through the process of transcription and translation. In the case studied above, the cell is directly responsible for the production of extra cellular proteins, and through internal cell metabolism, for the production of polysaccharides. The expression of the Urease in Bacillus is not fully understand, but scientists have found that urease can be induced in the presence of urea in 
other bacteria [36]. The cellular space can be manipulated through recombinant DNA and synthetic DNA approaches which either modify cells or attempt to create completely new cells. In our experiments, the radical difference in material outcomes from two similar but different cellular state spaces is demonstrated through the use of two different strains of bacteria.

The chemical state space is considered to be outside the cell, but is also in direct chemical interaction with the cell itself and (as in the case of a biofilm) can be created or modified by the cells themselves. In our biomineralization experiments, the chemical space was, initially, composed of urea which may trigger the production of urease in the cell, Calcium in the agar, and the presence of carbon in the air. Bacterial cells, however, progressively alter their chemical environment by releasing extra cellular materials such as Urease, which alter the $\mathrm{pH}$ of the chemical environment and induce mineralization.

Finally the physical state space constitutes the physical forces and energy within the environment. In the bacteria experiments described above, for example, this state space is defined by the physical confines of the agar plate, the temperature of the growth environment, and the physical structure of the agar itself. Again, the physical space is altered by the bacteria and its extra cellular processes. In the case of the $B$. pasteurii, the appearance of filaments seems to suggest a physical infrastructure which supports the distribution of crystals.

\subsection{The design framework for a synthetic shell}

Using the model described above we can develop a design framework which articulates the role of the designer in defining the state space of biological material construction. To help illustrate this framework, we can imagine a project in which a designer wants to create a synthetic shell consisting of a self-supporting three-dimensional structure composed of bio-induced calcium carbonate crystals, which are variegated through the shell's section to provide different material properties. In addition, we can use Oxman's model of 'design drivers' [37] to describe the different aspects of the process of material articulation in terms of fabrication, assembly, materiality and geometry. In envisaging a model for biological design, all of these processes are considered together in terms of their application to the design of a single object, but are also considered separately in terms of how they are defined in relation to specific methods of design. In the design of materials using biomineralization, these processes are descriptions of material scale (molecular fabrication, micro assembly, and macro geometry, Fig. 4) but, in addition, each is informed by an interrelated set of state spaces which may act at multiple scales.

Each of these processes can be influenced by the combination of the three state spaces, i.e. the cellular, chemical and physical. There is a transaction between each state space which is distinct for each scale of material production. The designer has the option of manipulating or constraining each state space, which in turn influences the organization and eventual behavior of the material assemblage. These options are played out for material, fabrication and assembly in diagram (Fig. 5(a)-(c)) for the synthetic shell example. What the diagram shows are the information exchanges between the physical, chemical and cellular state spaces which define each scale of construction. However, in each instance it is also possible to imagine different starting points and different ways of constraining either the cellular, chemical or physical state space or a combination. This is achieved by controlling information held within the system and constraining the space of interaction, as done in the examples described in Section 2.3. To illustrate the design implications of this in more detail, we can focus on geometry (Fig. 6) by considering how to induce a biological system to distribute calcium carbonate into a shell-like structure.
In the context of an abalone shell, as described above, the shell's shape and patterning are derived from a process of morphogenesis where soft tissues provide a surface on which biomineralization can occur. Morphogenesis refers to the process by which cells organize themselves into patterns. Studies in developmental biology have shown that the molecular pathways, which enable cells to interact with one another in order to self-assemble, are highly complex often consisting of thousands of protein interactions [38] However, observations on the effects of cellular organization seem to suggest that cells are, at some level, steered by simple rules. Alan Turing in his seminal paper 'The Chemical Basis of Morphogenesis' [39] gave accounts of mathematical formulae which describe pattern generation of, for example, zebra stripes or account for the spacing of teeth. The correlation between the computational models and the observed characteristics of various biological morphologies is compelling but not proven. However, it has been proposed that, rather than attempting to bridge the gap between cellular complexity and observed morphological patterns we can, instead, design and program cells to reproduce behaviors which follow patterns found in Turing equations through much simpler cellular circuits. For example, Davies has presented some initial experiments to attempt to program a Turing pattern to occur in cell culture based on the genetic manipulation of cells such that they produce promoter and inhibitor molecules which, by altering the chemical gradients across a plate of connected (epithelial) cells, can cause a gene responsible for producing a luminescent chemical to be turned on and off in a phased pattern [40]. Davies has suggested that there are ten basic mechanisms of animal morphogenesis [41] (see Fig. 7) which describe how cells collaborate to pattern tissues in biological systems. In each case, cells communicate with one another to assemble and deform into three-dimensional structures.

Given this understanding of morphogenesis, along with the model of three state spaces, the designer would have a choice of material articulation strategies (illustrated in Fig. 6). A cellular approach would involve selecting and modifying the appropriate genes. The information would then be held within the genes and the cells would structure themselves into a desired form, such that a scaffold is formed on which the mineral crystals can be patterned. Alternatively, the designer could start by shaping the physical environment itself, in effect creating a physical mold, constrain the cell growth and promote mineralization on a predetermined surface. This involves constraining the state space of material production. The decision of which one of these approaches to adopt depends on the designer's intentions and the complexity of the state space of manipulation (recent approaches to this sort of biological fabrication are discussed in [42]). The genetic factors responsible for morphogenesis may, for example, be highly complex and well beyond the state of the art for genetic programming. This could suggest a physical approach whereby the appropriate scaffold would need to be built using physical fabrication in a material, which could act as a substrate for the cells and their chemical environments

Similar decisions would be made for each aspect of the material construction, i.e. fabrication, assembly, etc. The designer's choice in each instance is about what state space or combination of state spaces to manipulate to achieve the desired result, based on issues of complexity, technical viability and control.

\subsection{Experiment 2: the interconnected state-space of biomineraliza- tion}

The framework discussed above describes the design of biological materials and structures in terms of the state space of living cells, and their physical and chemical environment of construction. It is important to recognize that there are no clear 


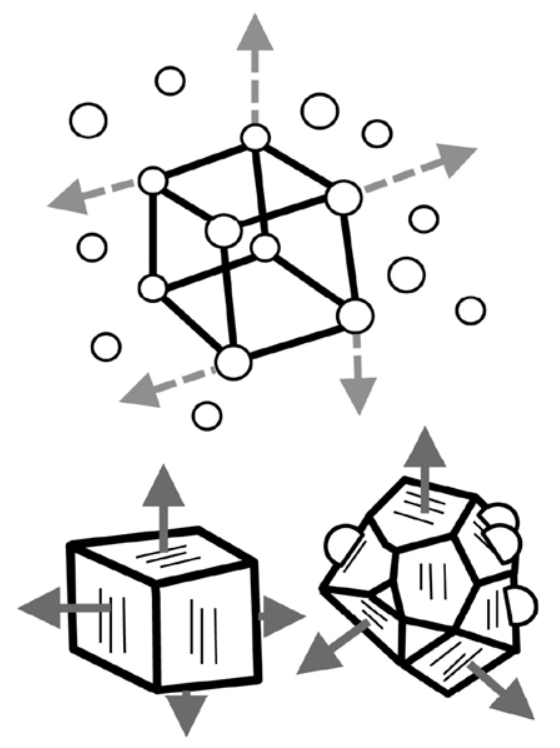

Material

Molecular level assembly of carbon and calcium atoms to create a calcium carbonate crystal.

Fabrication

The fabrication of individual crystals in the presence of extracellular materials (e.g. amino acids) to control crystal nucleation and morphology.
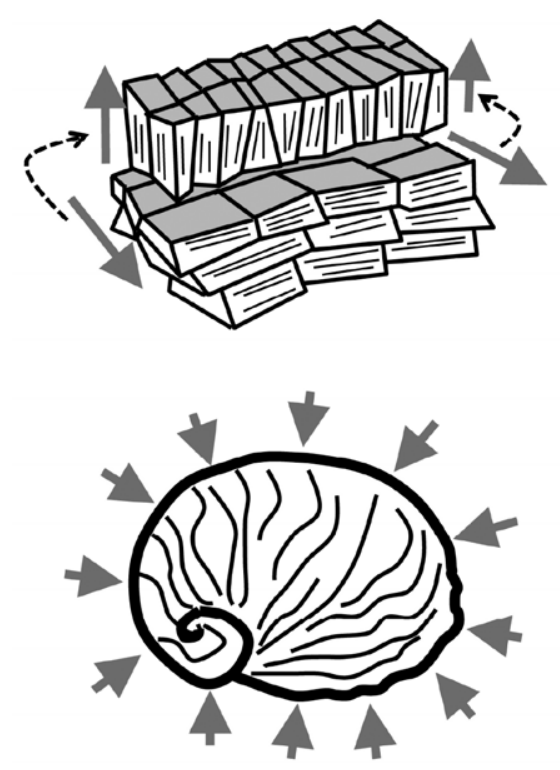

Assembly

The assembly of groups of morphologically distinct crystals in layers through the phased influence of extra cellular materials.

\section{Geometry}

The aggregation of calcium carbonate layers to form the three dimensional shape of the shell.

Fig. 4. Diagrams to illustrate the processes of material articulation in creating a calcium carbonate abalone shell at different scales of construction.

distinctions in real biological systems between, for example, material assembly, fabrication and shape. Furthermore, because each state space is interconnected, changing one has implications for the others. This interconnectedness and nonlinearity is implicit in a material ecological approach to design but, in the context of SB the state space also includes the possibility of altering the elements of material production at a molecular level, through genetic engineering. Each scale of material production has its own logic but they are also connected. Without explicitly engineering the living organism, the way in which genes are expressed can be altered by manipulating the environment.

To simply illustrate the effects of this multi-scale state space, we performed a short secondary experiment with a group of postgraduate architecture students to understand the implications on both geometry and crystal fabrication by specifically altering the physical state space for bacteria biomineralization.

The students worked on the design of a series of 'bioreactors' of increasing complexity, both in terms of the number of variables tested and the deviation from more traditional agar plate type experiments. Bioreactors are engineered devices which enable or support a biologically active environment. Three of the bioreactors involved modifying the conditions of a regular agar plate, and a fourth involved the use of a purpose built container. In all of the experiments, the emphasis was on controlling the physical conditions at the scale of their geometry. In the cases shown here, $B$. pasteurii was used in the same mineralization conditions (in terms of the chemical composition of the Agar and the temperature of the incubation period). In each case the bioreactor either provided a mold or a scaffold to hold the agar and promote the growth of the bacteria (see [43] for more extensive description of the experiment). Fig. 8 shows one of the simple bioreactors with instructions on the preparation of the agar substrate, and the locations in which the bacteria were to be inoculated. The cultures were incubated for twenty-four hours using both species of bacteria used in the earlier experiments. Upon completion, samples were collected and prepared for further analysis through Electron Microscopy.

The aim of the experiments was to create physical scaffolds which would impact on the growth of the bacteria and thus the 
a

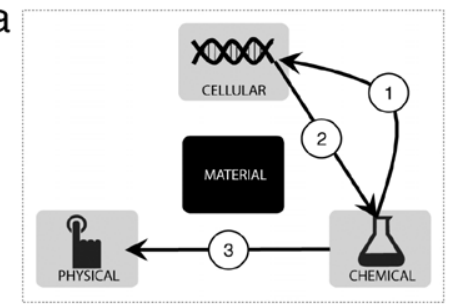

b

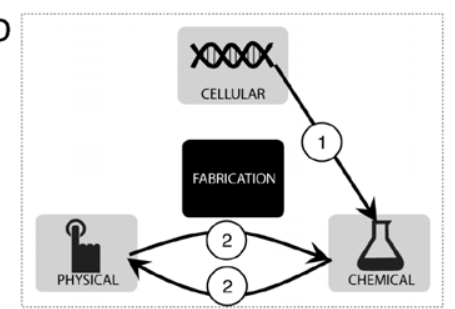

C

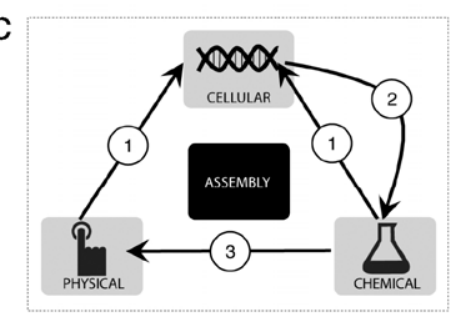

Material refers to the composition of the shell, in this case calcium carbonate (although we could imagine other biomineralization processes).

Process:

The presence of urea in the environment (1) causes urease (2) to be expressed by the cell and induces the formation of calcium carbonate as the $\mathrm{pH}$ of the environment is altered (3).

Fabrication refers to the process by which the individual crystals are assembled into particular structures and morphologies.

\section{Process:}

Compounds released by the cell (1) react with the surface of the forming crystal (2) to constrain its growth and geometry.

\author{
Assembly refers to the process by which the crystals \\ agglomerate into larger structures and differentiate the \\ morphology of the crystals on different layers (as in the \\ case of the abalone shell). \\ Process: \\ We can enable the cell to produce two phases of extra \\ cellular proteins which induce different crystal \\ morphologies. However there needs to be a trigger \\ either from the physical or the chemical (1) \\ environments to tell the cells to switch to a different \\ chemical phase (2) and build different crystal \\ structures (3).
}

Fig. 5. Diagrams to show the interaction between different 'state spaces' to create, fabricate and assemble calcium carbonate crystals.

Geometry refers to process leading to the eventual form of the shell as an aggregate of the crystals assembled together in a complete structure.

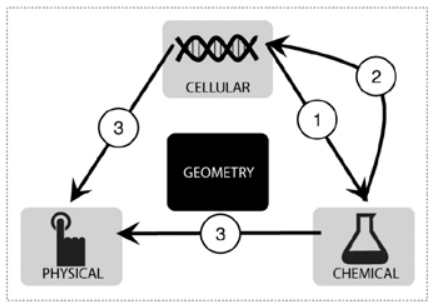

Process Option 1:

The cells communicate with each other through chemical signals (1) to self assemble through morphogenesis (2). This constrains both the physical shape of the cellular scaffold and the flow of calcium carbonate inducing materials (3).

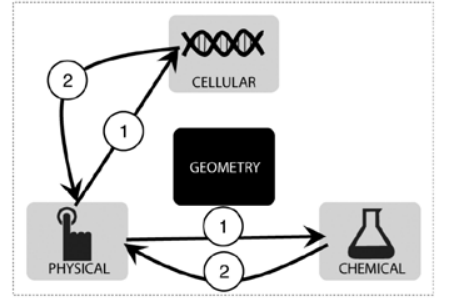

Process Option 2:

A physical constraint (e.g. a mold) is used to constrain the cellular growth (1) and the flow of extra cellular

materials (1) to a confined space (2).

Fig. 6. Diagrams to show two possible scenarios for the construction of the shells geometry, as an aggregate of calcium carbonate crystals.

distribution of crystals. This meant controlling the geometry of biomineralization by altering the physical parameters of the space. What we discovered, however, was that in addition to controlling the geometry in terms of the aggregate precipitation of the crystals, the morphology of the individual crystals was also changed. The clearest demonstrations of this effect can be seen in the electron microscope image in Fig. 9. This experiment consisted of an agar plate that had been modified to provide only thin channels into which the agar was poured (Fig. 8). The B. pasteurii was inoculated in a circular well at one end of the channel. As expected, crystals were distributed along the channel even millimeters away from the bacteria colony. However, compared to the earlier sample (Fig. 2) the crystal morphology is significantly different-in this case broken up into clusters of smaller crystals. The reason for this is unclear given that all other factors are the same (including bacteria strain, agar composition, and temperature). We can hypothesize, however, that altering the physical environment may have altered the diffusion of extra cellular substances, such that crystals had less time to grow (perhaps because the agar has tended to dry out more quickly) or that growth has been constrained (perhaps because the chemical diffusion has been limited by the narrow channel). In this case, the chemical and physical environment 


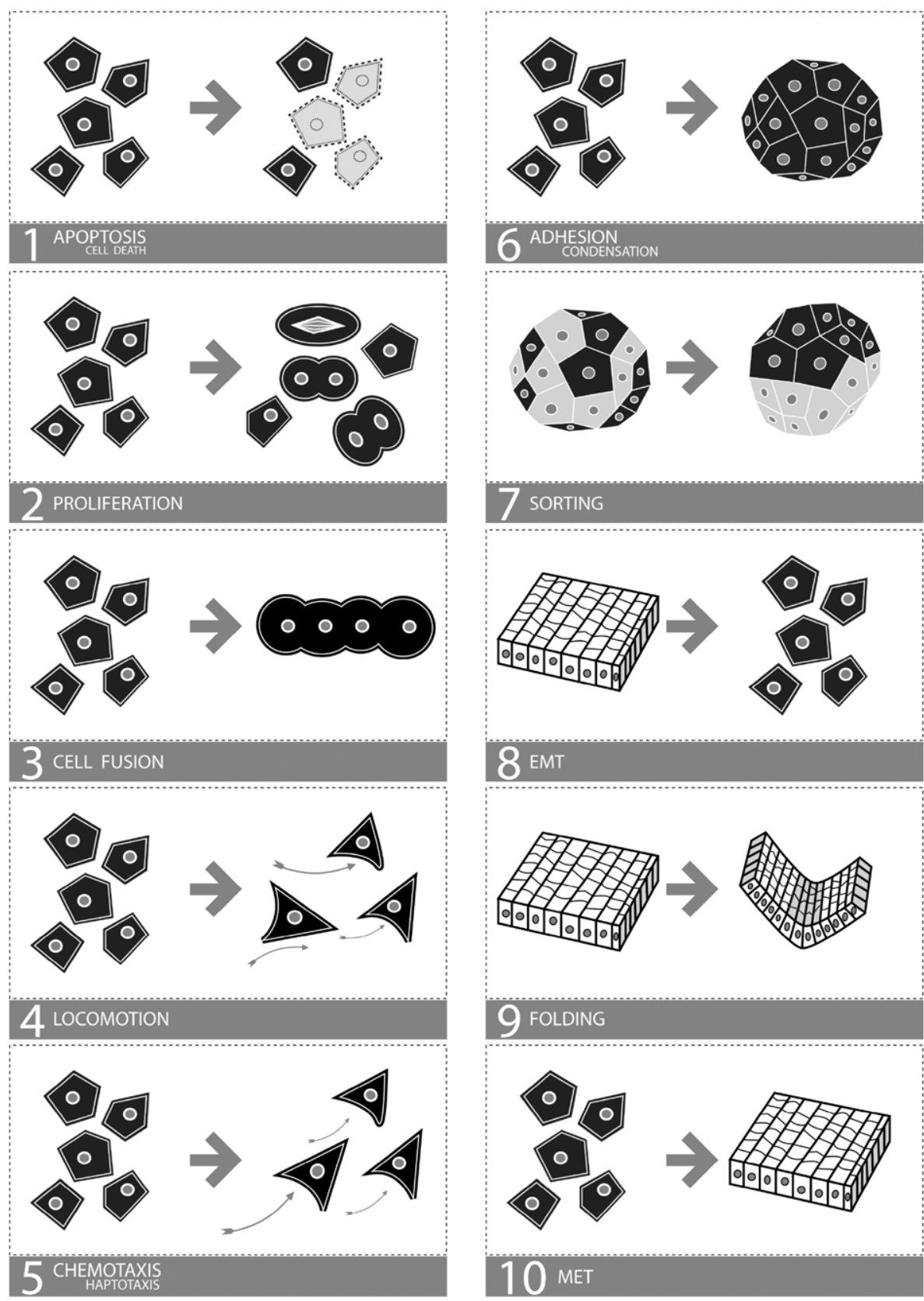

Fig. 7. The ten cellular mechanisms of morphogenesis based on the original diagram by Davies in [39].

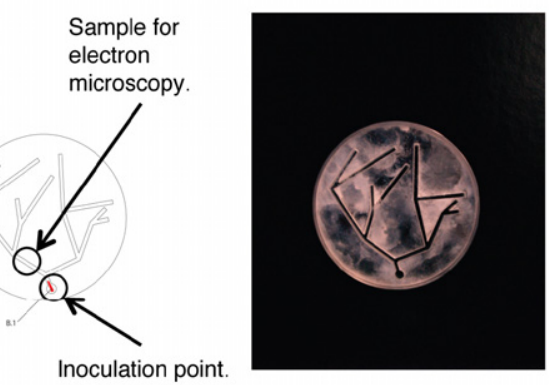

Agar plate filled with Perspex sheets with channels to constrain the growth of the bacterial and the precipitation of calcium carbonate samples.

Procedure:

1. UV sterilization of the mould before fixing in the agar plate.

2. Agar poured into the channels filling them but without overflowing.

3. Inoculate the bacteria in the circular well.

Fig. 8. An example of a bioreactor created to constrain the growth of bacteria and the precipitation of calcium carbonate crystals. The diagram shows the experimental protocol as well as the inoculation and sampling points on this modified agar plate. 

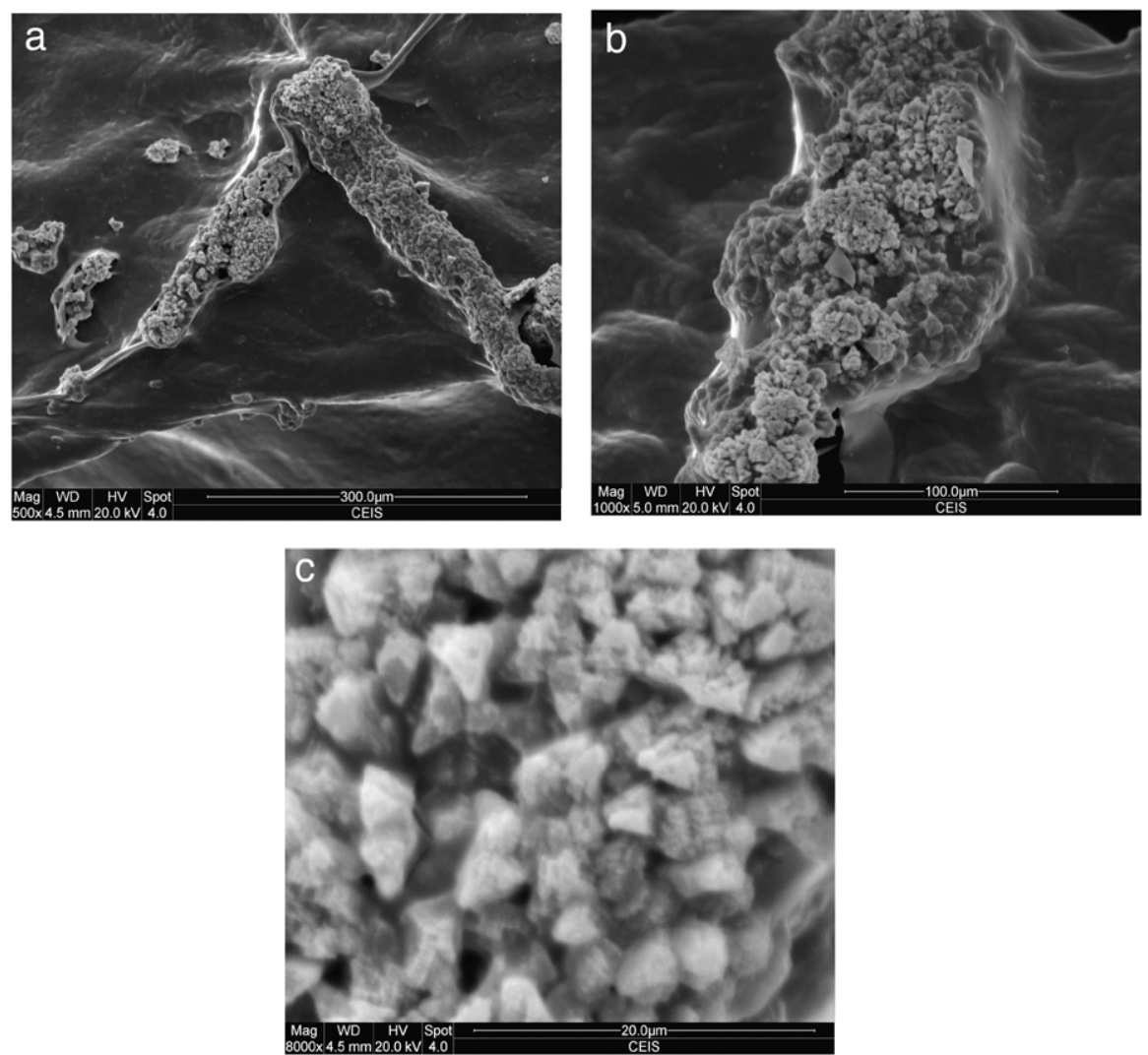

Fig. 9. Electron microscope images of the crystal forms taken from samples in the bioreactor shown in Fig. 8 .

of biomineralization, and thus the control of fabrication and geometry, is shown to be strongly interrelated, even when the intension of this design exercise was to only influence the geometry of the material assemblage.

\section{Conclusion}

In considering the role of SB in the creation of new types of material, we have provided a grounded speculation on, not only the possible outcomes of an SB approach to material production, but also a new set of design processes. These experiments are clearly limited and, although illustrative, only hint at the challenges which need to be overcome in developing a material system with SB. Many of the processes being observed are not yet fully understood and are difficult to assimilate in a design process. They have, however, shown that, in the case of bacterially induced biomineralization, nothing in the DNA of B. pasteurii, for example, encodes explicitly the faceted spherical crystals of calcium carbonate. We therefore suggest that, whilst SB emphasizes the primacy of DNA in the creation and control of all biological systems, the state space of biological material construction is composed of different interrelated conditions and scales of interaction, each of which can be manipulated independently.

Beyond the potential application domains of these new material possibilities, the notion of engineering nature encourages designers to think in ways which are unfamiliar in relation to how the material world is and might be shaped. Early conceptualizations of SB as an engineering design discipline invoke a limited view of biological systems. Whilst inert materials are amenable to direct manipulation by the hands of the crafts person, biological systems are subject to more indirect control or influence. In turn, the effects of such biological systems on material processes, such as mineralization, are also conducted under the indirect influence of biological processes through both physical and chemical control of the crystal's state space. While this way of thinking about material articulation appears alien to design practice, the broader notion of state spaces of influence is not new to design discourse. It follows that a material ecology approach, which understands the interrelation between material, fabrication and assembly in the context of a dynamic reciprocity, naturally lends itself to application in the design of real biological systems. The challenge for the designer of material using SB is to tackle two main issues. The first is concerned with how morphology is evolved through complex interactions across scales and state spaces. The second is the need to develop the appropriate tools and methods of abstraction and representation to design such systems prior to the actual biological engineering, whilst at the same time understanding the emergent complexity which will inevitably challenge their design intentions. In essence, SB and the material practice derived from it represent the ultimate test of a material ecological approach to design.

\section{Acknowledgments}

We would like to thank Newcastle University and Northumbria University for their support in this project. We would also like to thank John Beattie. Malcolm Welford, Alex Lyon and Marcus Ryden for their work on this project.

\section{References}

[1] Andrianantoandro E, Basu S, Karig DK, Weiss R. Synthetic biology: new engineering rules for an emerging discipline. Mol Syst Biol 2006;2:1-14.

[2] Oldham P, Hall S, Burton G. Synthetic biology: mapping the scientific landscape. PLoS One 2012;7:e34368.

[3] Voigt C. Synthetic biology. London: Royal Academy of Engineering; 2012.

[4] Lloyd Jones P. ConText Messaging. 306090 2007;11: 33-8.

[5] Harris RF. Good news for mice! Curr Biol 1999;9:16-30.

[6] Khalil AS, Collins JJ. Synthetic biology: applications come of age. Nat Rev Genet 2010;11:367-79. 
[7] Endy D. Foundations for engineering biology. Nature 2005;438:449-53.

[8] Reardon S. Visions of synthetic biology. Science 2011;333:1242-3.

[9] Ginsberg A, Calvert J, Schyfter P, Elfick A, Endy D. Synthetic aesthetics: investigating synthetic biology's designs on nature. Cambridge (Mass): MIT Press; 2014.

[10] Purnick PEM, Weiss R. The second wave of synthetic biology: from modules to systems. Nature Rev Mol Cell Biol 2009;10:410-22.

[11] Isalan M, Lemerle C, Michalodimitrakis K, Horn C, Beltrao P, Raineri E, et al. Evolvability and hierarchy in rewired bacterial gene networks. Nature 2008; 452:840-5.

[12] O'Malley Ma. Making knowledge in synthetic biology: design meets kludge. Biol Theory 2009;4:378-89.

[13] Haraway DJ. Crystals, fabrics and fields: metaphors that shape embryos. Berkeley: North Atlantic Books; 2004.

[14] Gilbert SF, Sarkar S, Hertwig O, Spemann H, Harrison R, Dalq AM, et al. Embracing complexity? Organicism for the 21st Century. Dev Dyn 2000;9:1-9.

[15] Oxman N, Rosenberg JL. Material-based design computation an inquiry into digital simulation of physical material. Int J Archit Comput 2010;05:25-44.

[16] Hensel M. (Synthetic) life architectures: ramifications and potentials of a literal biological paradigm for architectural design. Archit Des 2006;76:18-25.

[17] Cruz BM, Pike S. Neoplasmatic design. Archit Des 2008;76:6-7.

[18] Spiller N, Armstrong R. Its a brand new morning. Archit Des 2011;14-25.

[19] Hanczyc MM, Ikegami T. Protocells as smart agents for architectural design. Technoetic Arts A J Specul Res 2009;7:117-20.

[20] De Wolf T, Holvoet T. Emergence versus self-organisation: different concepts but promising when combined. Eng self-Organ. syst 2005;1-15.

[21] Griffith S, Goldwater D, Jacobson JM. Self-replication from random parts. Nature 2005;437:636

[22] Griffith S. Growing machines. MIT; 2004.

[23] Tibbits S, Cjeung K. Programmable materials for architectural assembly and automation. Assem Autom 2012;32:216-25.

[24] Lin AY-M, Chen P-Y, Meyers MA. The growth of nacre in the abalone shell. Acta Biomater 2008;4:131-8.

[25] Mann S. Biomineralization: principles and concepts in bioinorganic materials chemistry. Oxford: Oxford University Press; 2001.

[26] Mann K, Siedler F, Treccani L, Heinemann F, Fritz M. Perlinhibin, a cysteine, histidine-, and arginine-rich miniprotein from abalone (Haliotis laevigata) nacre, inhibits in vitro calcium carbonate crystallization. Biophys J 2007;93: 1246-54.

[27] Stocks-Fisher S, Galinat J, Bang S. Microbiological precipitation of $\mathrm{CaCO}_{3}$. Soil Biol 1999;31:1563-71.

[28] Tice MM, Thornton DCO, Pope MC, Olszewski TD, Gong J. Archean microbial mat communities. Annu Rev Earth Planet Sci 2011;39:297-319.

[29] Jonkers HM. Self healing concrete? A biological approach, 2007. p. 195-204.

[30] Cracks in your concrete? You need BacillaFilla n.d http://www.ncl.ac.uk/press.office/press.release/item/cracks-in-yourconcrete-you-need-bacillafilla\#.UY5Lib9Z-8 [accessed 11.02.2014]

[31] Hammes F, Boon N, Villiers JDe, Siciliano SD, Verstraete W. Strain-specific ureolytic microbial calcium carbonate precipitation. Appl Environ Microbio 2003;69.

[32] Belcher A, Wu X, Christensen R. Control of crystal phase switching and orientation by soluble mollusc-shell proteins. Nature 1996;381:56-8.

[33] Wheeler AP, George JW, Evans CA. Control of calcium carbonate nucleation and crystal growth by soluble matrix of oyster shell. Science 1981;212:1397-8.

[34] Braissant O, Cailleau G, Dupraz C, Verrecchia EP. Bacterially induced mineralization of calcium carbonate in terrestrial environments: the role of exopolysaccharides and amino acid. J Sediment Res 2003;73.

[35] Ercole C, Bozzelli P, Altieri F, Cacchio P, Del Gallo M. Calcium carbonate mineralization: involvement of extracellular polymeric materials isolated from calcifying bacteria. Microsc Microanal 2012;18:829-39.

[36] Wyborn NR, Scherrt DJ, Jones CW. Purification, properties and heterologous expression of formamidase from Methylophilus methylotrophus. Mirobiology 1994; 140:191-5.
[37] Oxman N. Get real: towards computational geometry. Int J Archit Comput 2007;05:663-84.

[38] Von Mering C, Krause R, Snel B, Cornell M, Oliver S, Fields S, et al. Comparative assesment of large-scale data sets of protein-protein interactions. Nature 2002;217:399-403.

[39] Turing AM. The chemical basis of morphogenesis. Philos Trans R Soc Lond 1952;237:37-72

[40] Davies J. Synthetic biology approaches to Turing patterns 2012. https://www.youtube.com/watch?v=20GsPPENvQA [accessed 11.02.2014].

[41] Davies J. Synthetic morphology: prospects for engineered, self-constructing anatomies. J Anat 2008;212:707-19.

[42] Lutolf M, Hubbell J. Synthetic biomaterials as instructive extracellular microenvironments for morphogenesis in tissue engineering. Nature Biotechnol 2005;23:47-55.

[43] Dade-Robertson M, Zhang M, Ramirez-Figueroa C, Hernan L, Beattie J, Lyon A, et al. Proto-materials: material practices in architecture at molecular and cellular scales. In: Stacey M, editor. Prototyp. archit.. London: Building Centre; 2013. p. 211-22

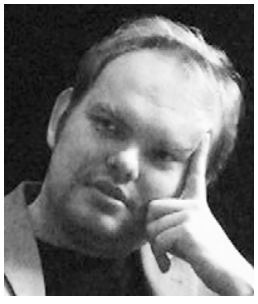

Martyn Dade-Robertson is Lecturer in Architecture in the School of Architecture Planning and Landscape at Newcastle University. Martyn originally did a degree in Architecture at Newcastle before embarking on an M.Phil. and Ph.D. at Cambridge University (Darwin College) with a theses Entitled 'Information Architecture in Screen Based Semantic Spaces'. This Ph.D. research led to a number of successful cross disciplinary projects and collaborations, including an internship at Microsoft Research and the book "The Architecture of Information", published by Routledge in 2012 .

His most recent research has been into the role of Synthetic Biology and Architectural design with an emphasis on material practices and prototypes.

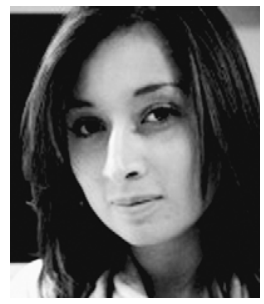

Carolina Ramirez Figueroa is a doctoral student at Newcastle University working on the intersection of Architecture, Synthetic Biology and Digital Technologies. Carolina has a multi-disciplinary background. After completing one year in Product Design, Carolina moved on to train as a full architect and later studied her Master's degree in Digital Architecture at Newcastle University. Her degree dissertation, entitled Synthetic Morphology and Dynamic systems in Architecture, looks at developing a mediating artifact that accounts for biological rules of morphogenesis. Her current research interests concern with the architectural immanence of morphogenesis and deal with conflicts of scale in biological systems. Carolina is currently researching bacterial patterning.

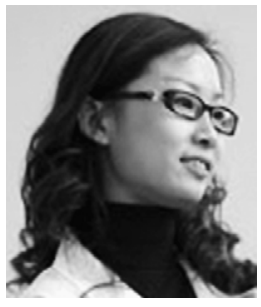

Meng Zhang studied for her B.Sc. in Biotechnology at ShanDong Agriculture University, China. In the UK she was then rewarded Overseas Research Scholarship by UK Universities and studied for a Ph.D. in Proteomics in Northumbria University. Meng is currently a lecturer at Northumbria University where her research interests are on Proteomics and Metabolomics study of pathogens and Synthetic Biology on Biocatalysis. Meng has published widely on Molecular Biology and Microbiology and is currently investigating wider applications of Synthetic Biology. 